\title{
Vergessene Kooperationen
}

\section{Pascal Germann}

Dr. phil., Institut für Medizingeschichte der Universität Bern

Die Humangenetik stellt heute zweifellos eine Leitdisziplin innerhalb der Medizin dar. Die zentralen Methoden der heutigen Humangenetik bildeten sich indessen spät heraus. Das Gebiet der humanen Zytogenetik erlebte erst Ende der 1950er Jahre einen fulminanten Aufstieg, und bis zur Herausbildung der molekularen Humangenetik dauerte es ein weiteres Jahrzehnt. In Lehr- und Handbüchern der Humangenetik wird denn auch gemeinhin auf die sehr junge Geschichte der Humangenetik verwiesen. Erst nach 1945 habe sich die Humangenetik zu einer eigentlichen Wissenschaft entwickelt und sich innerhalb der Medizin als eigenständiges Forschungs- und Praxisfeld etabliert. Eine solche Darstellung blendet aus, dass das Studium der menschlichen Vererbung bereits in der ersten Hälfte des 20. Jahrhunderts zu einem wichtigen Forschungsbereich der Medizin aufgestiegen war. Ausgeklammert bleibt dabei auch ein dunkles Kapitel der Geschichte der Humangenetik: Viele prominente Figuren der frühen Humangenetik waren in der eugenischen Bewegung engagiert, und die meisten Humangenetiker teilten die Grundüberzeugung der Eugeniker, dass Massnahmen erforderlich seien, um "genetisch hochwertigen» Nachwuchs zu fördern und "genetisch minderwertigen" Nachwuchs zu verhindern. Auch für die Schweiz lässt sich zeigen, dass die Geschichte der Humangenetik untrennbar mit derjenigen der Eugenik verbunden ist. Meine Forschungen zu den Anfängen der schweizerischen Humangenetik weisen zudem auf einen weiteren Zusammenhang hin, an den ungern erinnert wird: Der Aufstieg der humangenetischen Forschung erfolgte in engem Austausch mit der Wissenschaft und Eugenik in Nazideutschland. Dies soll nun am Beispiel des Zürcher Humangenetikers Ernst Hanhart verdeutlicht werden.

\section{Hanhart gilt als der erste Mediziner in der Schweiz, der sich vollständig auf die Human- genetik spezialisierte.}

Hanhart gilt als der erste Mediziner in der Schweiz, der sich vollständig auf die Humangenetik spezialisierte. Obwohl er nie einen Lehrstuhl besetzte, erlangte er sowohl in der Schweiz als auch international ein beachtliches wissenschaftliches Renommee und trug wesentlich zur Etablierung der Humangenetik in der

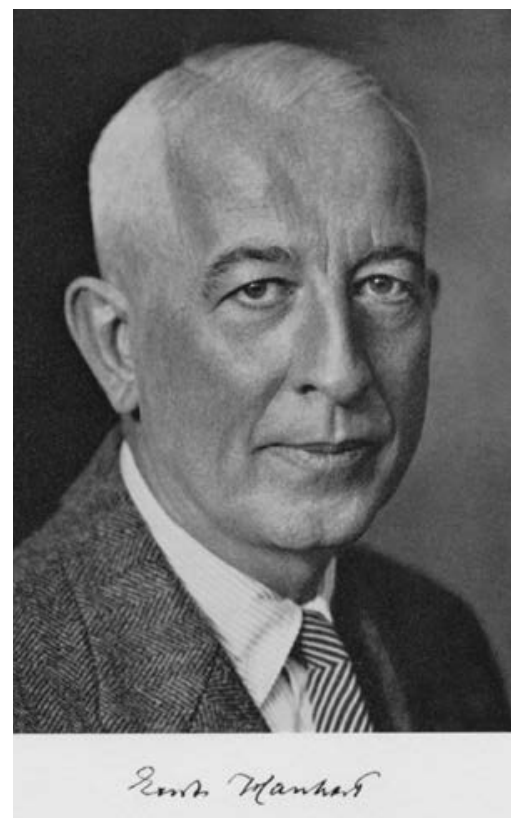

Ernst Hanhart (aus dem Jahresbericht der Universität Zürich, 1973/74).

Schweiz bei. Sein Ansehen gewann Ernst Hanhart insbesondere durch ein ehrgeiziges Forschungsprojekt, das er um 1920 initiierte und bis Ende der 1960er Jahre fortsetzte. Gemeinsam mit seinen Mitarbeitern durchforschte er eine Vielzahl alpiner Dörfer und sammelte riesige Mengen an genealogischen, demographischen und medizinischen Daten. Aus diesen Daten konstruierte Hanhart grosse Stammbäume, die er mendelistisch auswertete, um so die Entstehung und Verbreitung sowie die genetischen Übertragungswege von zahlreichen Pathologien zu untersuchen. Hinter seiner Forschungspraxis stand die Überlegung, dass in reproduktiv isolierten Populationen, wie sie in Schweizer Bergdörfern vermutet wurden, rezessiv vererbte Krankheiten und Anomalien häufiger manifest würden. Methodisch waren Hanharts genetische Feldstudien typisch für die mendelistische Vererbungsforschung seiner Zeit, die basales genealogisches Handwerk mit zunehmend elaborierten statistischen Methoden und modernen Diagnosetechniken verband. Aussergewöhnlich war indessen, wie umfassend Hanhart sein Programm der genetischen Bevölkerungserhebungen in die Praxis umsetzte. Hanhart 
wies seine Mitarbeiter jeweils an, die Verwandtschaftsund Abstammungsverhältnisse der Gesamtbevölkerung möglichst vollständig zu erheben und sämtliche Krankheiten, Anomalien und Missbildungen jedes einzelnen Einwohners zu registrieren. Bis 1960 führten Hanhart und seine Mitarbeiter in über fünfundzwanzig Dörfern und Gemeinden solche umfassenden «erbbiologischen Bestandesaufnahmen» durch, wie die Untersuchungen damals bezeichnet wurden.

\section{Seine genetischen Erkenntnisinteressen verband Hanhart von Anfang an mit eugeni- schen Zielsetzungen.}

Seine genetischen Erkenntnisinteressen verband Hanhart von Anfang an mit eugenischen Zielsetzungen: So sollten seine Bevölkerungserhebungen auch Risikogruppen identifizieren, die es ständig zu beobachten galt. Bezeichnenderweise wurden Hanharts Forschungen weitgehend von einer Zürcher Stiftung finanziert, die sich den Zielen der Eugenik bzw. der Rassenhygiene verschrieben hatte, wie der damals übliche Terminus im deutschsprachigen Raum lautete. Gemeint ist die 1921 gegründete Julius Klaus-Stiftung für Vererbungsforschung, Sozialanthropologie und Rassenhygiene. Aufgrund ihrer grosszügigen Finanzierung genetischer Forschungen leistete die Stiftung einen wesentlichen Beitrag zur Entwicklung der Humangenetik in der Schweiz. Auch für Hanharts Karriere war die Förderung durch die Stiftung von zentraler Bedeutung. Nur dank ihren regelmässigen Subventionen konnte Hanhart sein Forschungsprogramm so ambitioniert in die Praxis umsetzen.

Als Ernst Hanhart im Jahr 1927 in «Vererbungs- und Konstitutionslehre» an der Medizinischen Fakultät der Universität Zürich habilitierte, galt sein Arbeitsgebiet als noch wenig bedeutend. Ein Habilitationsgutachter vermerkte beispielsweise kritisch, dass Hanhart «nur einen ganz schmalen Sektor der inneren Medizin» pflege. ${ }^{1}$ Als der Zürcher Vererbungsforscher fünfzehn Jahre später zum Titularprofessor ernannt wurde, gab es keine solchen Vorbehalte mehr. Die Gutachten verwiesen nicht nur auf die wissenschaftlichen Leistungen und das internationale Ansehen Hanharts, sondern betonten darüber hinaus, dass die Verleihung der Professur «auch aufgrund der Wichtigkeit seines Fachgebietes» begründet sei, das «neuartige Erkenntnisse über das Wesen zahlreicher Krankheiten» ermögliche. ${ }^{2}$ Dementsprechend bestand im Jahr 1942 innerhalb der Medizinischen Fakultät ein Konsens, dass die «Erbforschung in der Medizin eine grosse Bedeutung erlangt» habe. ${ }^{3}$ Innerhalb eines kurzen Zeitraums kam es folglich zu einem bedeutenden
Aufschwung und Prestigegewinn der humangenetischen Forschung in der Schweiz.

Eine wesentliche Rolle beim Aufstieg der medizinischen Vererbungsforschung in den 1930er Jahren spielte ein transnationaler Kontext. Im Jahr 1933 kam in Deutschland ein Regime an die Macht, das eugenische Forderungen in einer bislang unerreichten Radikalität in die Praxis umsetzte. Bereits 1933 verabschiedete das Kabinett Hitlers das «Gesetz zur Verhütung erbkranken Nachwuchses», das die Anwendung von direkter Gewalt sanktionierte, um als «erbkrank» geltende Personen zwangsweise unfruchtbar zu machen. Vor diesem Hintergrund kam es in Nazideutschland zu einer eigentlichen "Symbiose» zwischen humangenetischer Forschung und staatlicher Rassenhygiene. ${ }^{4}$ Zum einen taten sich die meisten deutschen Humangenetiker mit viel Eigeninitiative hervor, um ihr Know-how in den Dienst der nationalsozialistischen Rassenhygiene zu stellen. Zum anderen erhielten Humangenetiker ein beispielloses Ausmass an staatlicher Unterstützung und politischer Aufmerksamkeit. Die Politik des neuen Regimes versprach neue Forschungs- und Karrieremöglichkeiten. Hitlers Machtergreifung veränderte auch die Bedingungen, unter denen sich die humangenetische Forschung in der Schweiz entwickelte. Zwar distanzierten sich einige Wissenschaftler in der Schweiz von der nationalsozialistischen Rassenhygiene, doch im Bereich der Humangenetik war die Bereitschaft gross, den Bedeutungsgewinn ihres Forschungsgebiets im nördlichen Nachbarland als Chance zu nutzen. Mit der Machtergreifung der Nationalsozialisten eröffnete sich auch für Hanhart ein neuer Resonanzraum, den er auszunutzen bestrebt war. Dies verdeutlicht seine $\mathrm{Pu}$ blikationstätigkeit, die er während der Nazizeit stark auf Deutschland ausrichtete. Vor 1933 hatte er einen

\section{Hitlers Machtergreifung veränderte auch die} Bedingungen, unter denen sich die humangenetische Forschung in der Schweiz entwickelte.

einzigen Artikel in einer nichtschweizerischen Zeitschrift veröffentlicht. Demgegenüber publizierte er zwischen 1934 und 1939 mindestens zwölf Aufsätze in deutschen Fachzeitschriften, darunter auch in den zentralen Organen der nationalsozialistischen Rassenhygiene wie der 1934 gegründeten Zeitschrift «Der Erbarzt». In seinen Publikationen bemühte sich Hanhart, sein Forschungsprogramm an Erkenntnis- und Politikinteressen der deutschen Rassenhygiene anzuschliessen. So fokussierte er in seinen Beiträgen für deutsche Zeitschriften mitunter explizit auf Problemstellungen, die sich durch die Umsetzung der rassenhygienischen 
Gesetze in Nazideutschland ergaben. In einer umfangreichen Arbeit zur sogenannten Taubstummheit betonte Hanhart beispielsweise, dass seiner Forschung auch "grösste praktische Bedeutung" zukomme, weil das Gesetz zur Verhütung erbkranken Nachwuchses die Sterilisierung aller Träger der «vererbten Taubstummheit» verlange. ${ }^{5}$ Hanhart war somit gewillt, das nationalsozialistische Zwangssterilisationsprogramm mit handlungsrelevantem, medizinisch-genetischem Wissen zu unterfüttern.

\section{Ernst Hanhart gelang es nach 1945, sich} forschungsstrategisch umzuorientieren.

Vor diesem Hintergrund gewann der Schweizer Forscher erhebliches Ansehen in Deutschland. Davon zeugt, dass er 1938 in die Deutsche Akademie der Naturforscher Leopoldina aufgenommen wurde. Einen Höhepunkt erreichte Hanharts wissenschaftliches Engagement in Deutschland im Jahr 1940, als er gemeinsam mit Günther Just und anderen deutschen Wissenschaftlern ein fünfbändiges Werk mit dem Titel «Die Grundlagen der Erbbiologie des Menschen» herausgab. ${ }^{6}$ Es handelte sich dabei um das international erste umfassende Kompendium der Humangenetik. Für die deutsche Wissenschaft stellte das Werk zweifellos ein Prestigeprojekt dar, das die Führungsrolle der deutschen Humangenetik und zugleich deren Internationalität unterstreichen sollte. So betonte Just im Vorwort, dass auch "hervorragende ausserdeutsche Forscher» mitgearbeitet hätten. Gemeint waren damit in erster Linie Schweizer Genetiker, die dem Werk den Nimbus internationaler Wissenschaft verliehen: Von den insgesamt neun nichtdeutschen Autoren waren nicht weniger als sechs Schweizer.

Die engen Beziehungen, die deutsche Forscher zu Ernst Hanhart pflegten, wurden auch von politischer Seite unterstützt, wie folgendes Beispiel zeigt. Im Juli 1936 fand im holländischen Scheveningen die zwölfte Konferenz der "International Federation of Eugenic Organizations" statt. Hochrangige Vertreter der deutschen Rassenpolitik schrieben der Konferenz grosse Bedeutung für den internationalen Ruf der nationalsozialistischen Rassenhygiene zu. An der Konferenz sprach auch Ernst Hanhart, der über seine genetischen Erhebungen in den Schweizer Alpentälern berichtete. Die deutschen Vertreter waren von seinem Referat begeistert. In einem internen Bericht über die Konferenz, der für politische Stellen wahrscheinlich für das Reichsinnenministerium - verfasst war, wurde Hanharts Beitrag als einziger Vortrag eines nichtdeutschen Teilnehmers besonders positiv hervorgehoben: "Allgemeines Interesse fanden die gründlichen Stammbaum-Demonstrationen von Hanhart, Zürich, der wärmstens für die eugenischen Massnahmen in Deutschland eintrat und dessen mit unendlicher Mühe zusammengestellte und mit ärztlichen Diagnosen versehenen Verwandtschaftstafeln der Nachahmung in Deutschland nur wärmstens empfohlen werden kann. ${ }^{7}$ Wie der Bericht weiter festhielt, seien nämlich solche «systematischen erbbiologischen Bestandesaufnahmen eine wichtige Vorbedingung» zur Umsetzung der rassenhygienischen Gesetzgebung in Deutschland. Im Jahr 1943 finanzierte das Reichsinnenministerium denn auch einen Forschungsaufenthalt von Günther Just, um sich in der Schweiz mit Genetikern auszutauschen und insbesondere Ernst Hanharts Forschungspraxis der «erbbiologischen Bestandesaufnahmen" aus eigener Anschauung kennenzulernen.

Es gibt keine Indizien, dass Hanharts enge Verbindungen zur deutschen Rassenhygiene sich negativ auf sein akademisches Weiterkommen in der Schweiz ausgewirkt hätten. Als der Zürcher Regierungsrat Hanhart im Jahr 1942 den Professorentitel verlieh, wurde in der Begründung die Zusammenarbeit mit deutschen Humangenetikern vielmehr positiv hervorgehoben: «Von seinem [Hanharts] internationalen Ansehen zeugen seine Referate an internationalen wissenschaftlichen Tagungen und die Tatsache, dass er zu der von Prof. G. Just erstmals unternommenen Herausgabe eines grossen Handbuches über Erbbiologie als Mitherausgeber und Bearbeiter mehrerer Kapitel herangezogen worden ist. ${ }^{8}$ Die akademischen Lorbeeren, die Hanhart in Nazideutschland holte, nutzten ihm offensichtlich auch für seine wissenschaftliche Reputation in der Schweiz.

\section{In der Schweiz wurde Hanhart bald schon als bedeutender Pionier und Promotor der Humangenetik verehrt.}

Nach dem Krieg etablierten Humangenetiker ein Fachnarrativ, das erzählt, wie die schweizerische Humangenetik sich in Abgrenzung zur politisch korrumpierten Forschung in Nazideutschland herausgebildet habe. Dieses Narrativ, das sich bis heute hartnäckig hält, zeugt von einer bemerkenswerten Amnesie. Wie am Beispiel von Ernst Hanhart gezeigt, erfolgte der Aufstieg der Humangenetik in der Schweiz weniger in Abgrenzung als in regem Austausch mit der deut schen Vererbungsforschung und Rassenhygiene. Die Beziehungen schufen dabei eine Win-win-Situation. Zum einen trugen Schweizer Forscher zur Akzeptanz der nationalsozialistischen Erbgesundheitspolitik bei und untergruben Bestrebungen, die deutsche Human- 
9 Schweizerisches Bundesarchiv: CH-BAR\#E9510. 10\#1987/32\#558*, Franceschetti an Bundesrat H.-P. Tschudi, Präsident der Marcel Benoist-Stiftung, 12.7.1962.

Korrespondenz: Dr. phil. Pascal Germann Institut für Medizingeschichte der Universität Bern

Bühlstrasse 26

CH-3000 Bern 9

pascal.germann[at]

img.unibe.ch genetik international zu isolieren. Zum anderen profitierten Schweizer Humangenetiker von neuen Publikations- und Kooperationsmöglichkeiten, die sich aufgrund der Förderung der Vererbungsforschung in Deutschland auftaten. Insgesamt lässt sich die These erhärten, dass die Wissenschaftsbeziehungen zu Nazideutschland nicht unwesentlich zum Aufschwung der Humangenetik in der Schweiz beitrugen. Allerdings ist es auffallend, dass die auch mit Deutschland eng verbundenen Schweizer Humangenetiker keine offenen Sympathien zum Nationalsozialismus bekundeten. Damit knüpften sie den Fortbestand ihrer wissenschaftlichen Glaubwürdigkeit nicht an die Zukunft des Nationalsozialismus, sondern liessen sich forschungsstrategisch alle Optionen offen. Dies erleichterte den Anschluss an die internationale Humangenetik in der Nachkriegszeit. Ernst Hanhart gelang es jedenfalls nach 1945, sich forschungsstrategisch umzuorientieren. Er knüpfte neue Beziehungen zu der nun angelsächsisch dominierten Forschungsgemeinschaft, und seine Erhebungen in den alpinen Dörfern galten in der Nachkriegszeit als wichtiger Beitrag zur genetischen
Isolatforschung, die einen Boom erlebte. In der Schweiz wurde Hanhart bald schon als bedeutender Pionier und Promotor der Humangenetik verehrt. Der bekannte Genfer Augenmediziner Adolphe Franceschetti, der eine zentrale Rolle bei der Institutionalisierung der Humangenetik in der Nachkriegszeit spielte, schrieb 1962 schlicht: «Es ist dank der wissenschaftlichen Verdienste von Professor Hanhart, dass die Schweizer Genetik heute ein so grosses internationales Ansehen geniesst.» ${ }^{9}$

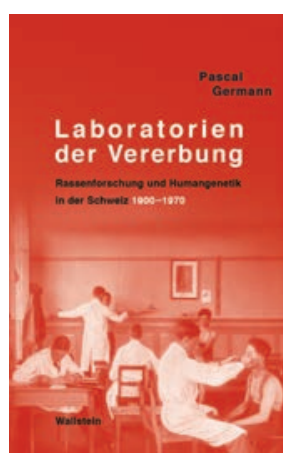

\section{Hinweis}

Der Beitrag basiert auf der jüngst erschienenen historischen Dissertation des Autors: Laboratorien der Vererbung. Rassenforschung und Humangenetik in der Schweiz, 1900-1970. Göttingen: Wallstein Verlag, 2016. 490 S., 54.00 €. ISBN: 978-3-83531950-9. Die Studie ist kürzlich mit dem Jahrespreis der Philosophischen Fakultät der Universität Zürich ausgezeichnet worden. 